DOI: 10.12957/demetra.2016.15564

\title{
Associação entre medidas antropométricas e de composição corporal com os componentes da síndrome metabólica e índice de qualidade da dieta em adultos com excesso de peso
}

\section{Association between anthropometric measurements and body composition with components of the metabolic syndrome and quality index diet in overweight adult individuals}

\author{
Adriana Moura Vieiral \\ Arthur da Silva Gomes \\ Renata Adrielle Lima Vieira \\ Fernanda Cacilda Silva ${ }^{2}$ \\ Helena Dória Ribeiro de Andrade Previato ${ }^{3}$ \\ Ana Carolina Pinheiro Volp ${ }^{4}$ \\ 1 Universidade Federal de Ouro Preto, Programa \\ de Pós-Graduação em Saúde e Nutrição da Escola \\ de Nutrição. Ouro Preto-MG, Brasil. \\ 2 Universidade Federal de Ouro Preto, Laboratório \\ de Fisiologia Cardiovascular, Departamento de \\ Ciências Biológicas, Instituto de Ciências Exatas e \\ Biológicas. Ouro Preto-MG, Brasil. \\ ${ }^{3}$ Universidade Estadual de Campinas, \\ Departamento de Alimentos e Nutrição, Faculdade \\ de Engenharia de Alimentos. Campinas-SP, Brasil. \\ ${ }^{4}$ Universidade Federal de Ouro Preto, \\ Departamento de Nutrição Clínica e Social, Escola \\ de Nutrição. Ouro Preto-MG, Brasil. \\ Correspondência / Correspondence \\ Ana Carolina Pinheiro Volp \\ E-mail:anavolp@gmail.com
}

\section{Resumo}

Introdução: $\mathrm{O}$ excesso de adiposidade, especialmente na região abdominal, associa-se a fatores de risco cardiovasculares como dislipidemia, diabetes mellitus e hipertensão arterial, e o padrão alimentar modula estas doenças crônicas. Objetivo: Avaliar a associação entre medidas antropométricas e de composição corporal com os componentes da síndrome metabólica (SM) e índice de qualidade da dieta (IQD) em indivíduos com excesso de peso. Métodos: Estudo transversal, realizado com indivíduos atendidos no Centro de Saúde da UFOP. Após a entrevista inicial, realizou-se avaliação antropométrica e foram solicitados exames bioquímicos. O consumo alimentar foi avaliado por instrumentos dietéticos, utilizados para calcular o IQD. As análises estatísticas foram realizadas no programa PASW 18.0. Foram utilizados o teste de Kolmogorov-Smirnov (para avaliar a normalidade dos dados) e a correlação de Pearson. Resultados: A prevalência de SM foi de $15,7 \%$. Houve associação entre o IQD com a concentração de colesterol total $(r=0,320 ; p=0,041)$, e associação inversa entre os níveis séricos de HDL-c com o índice de massa corporal $(\mathrm{r}=$ 0,434; $\mathrm{p}=0,002)$. A média de consumo dos componentes do IQD encontrou-se dentro das recomendações das Dietary Reference Intakes, exceto para o sódio, que estava acima, e o cálcio, que estava abaixo dos valores recomendados. Não houve diferença nos itens do IQD entre os indivíduos com presença e ausência de síndrome metabólica $(\mathrm{p}>0,05)$. Conclusão: Os resultados evidenciam acúmulo de gordura corporal, alteração dos parâmetros antropométricos e bioquímicos, ingestão inadequada de cálcio e 
de sódio, demonstrando a importância de um acompanhamento nutricional, prevenindo o surgimento de novas morbidades.

Palavras-chave: Índice de Qualidade da Dieta. Síndrome Metabólica. Parâmetros Antropométricos. Parâmetros Bioquímicos. Índice de Massa Corporal. Composição Corporal.

\section{Abstract}

Introduction: The adiposity excess, especially in the abdominal region, is associated with cardiovascular risk factors, such as dyslipidemia, diabetes mellitus type 2 and systemic arterial hypertension, among other clinical complications that affect quality of life. Aim: To evaluate the association between anthropometric measurements and body composition with components of metabolic syndrome (MS) and diet quality index (DQI) in subjects with overweight. Methods: Cross-sectional study conducted with patients treated at the UFOP Health Center. After the initial interview and anthropometric assessment, biochemical exams were requested. Dietary intake was assessed by dietary instruments used to calculate DQI. Statistical analysis were performed in SPSS 18.0 program. The Kolmogorov-Smirnov test (to evaluate the normality of the data) and the Pearson correlation were used. Results: The prevalence of MS was $15.7 \%$. There was association between the DQI score with serum total cholesterol $(\mathrm{r}=0.320 ; \mathrm{p}=0.041)$, and an inverse association between serum levels of HDL-c with body mass index $(r=-0.434 ; \mathrm{p}=0.002)$. The average consumption of the components of the DQI was within the recommendations of the Dietary Reference Intakes, except for sodium, which was above, and calcium, which was below of recommended values. There was no difference in the DQI items between individuals with and without MS ( $p>0.05)$. Conclusion: The results shows accumulation of body fat, changes in anthropometric and biochemical parameters, inadequate intake of calcium and sodium, demonstrating the importance of nutritional counseling, preventing the occurrence of new morbidities.

Key words: Diet Quality Index. Metabolic Syndrome. Anthropometric Parameters. Biochemical Parameters. Body Mass Index. Body Composition. 


\section{Introdução}

A prevalência de doenças crônicas não transmissíveis vem aumentando nas ultimas décadas, tornando-se um problema de saúde pública global, responsável por aproximadamente duas em cada três mortes no mundo. ${ }^{1}$ Dentre essas doenças, o sobrepeso e a obesidade tem sido alvo de grande preocupação, por serem fatores de risco para o desenvolvimento de outras morbidades crônicas. ${ }^{1,2}$

Segundo a Organização Mundial de Saúde (OMS), em 2014 mais de 1,9 bilhões de pessoas com 18 anos ou mais se encontravam acima do peso (39\%), sendo que destes, 600 milhões já eram considerados obesos (13\%). ${ }^{3}$ Em âmbito nacional, no ano de 2013, 50,8\% da população adulta estava acima do peso ideal e $17,5 \%$ eram considerados obesos. ${ }^{4}$

Assim, torna-se fundamental entender os fatores de risco envolvidos na gênese dessa doença, que abrange condicionantes biológicos, comportamentais, ambientais e genéticos. ${ }^{3}$ Nessa perspectiva, o sedentarismo e mudanças nos hábitos alimentares com o consumo cada vez maior de alimentos de alta densidade energética, vêm sendo considerados os fatores de risco modificáveis mais incisivos da incidência crescente da obesidade. ${ }^{5}$

Dessa forma, o governo conjuntamente com o Ministério da Saúde tem buscado implementar importantes estratégias políticas no enfrentamento dessas doenças. ${ }^{6}$ No intuito de incorporar mudanças nos hábitos alimentares, utilizam-se estratégias de intervenção nutricional e fazem uso de instrumentos que valorizam e incentivam a prática alimentar saudável. ${ }^{7}$

Para uma efetiva avaliação nutricional, além da antropometria, composição corporal e da análise bioquímica, a verificação do padrão de consumo de alimentos de uma população torna-se essencial. ${ }^{8}$ Ainda, devido à fácil aplicabilidade e ao baixo custo operacional, o índice de massa corporal (IMC) tem sido utilizado como um indicador de sobrepeso (IMC $\geq 25 \mathrm{~kg} / \mathrm{m}^{2}$ ) e obesidade $\left(\mathrm{IMC} \geq 30 \mathrm{~kg} / \mathrm{m}^{2}\right)$ em diversos estudos epidemiológicos envolvendo adultos. ${ }^{9}$

Quando se deseja estabelecer a quantidade de calorias, macronutrientes e micronutrientes ingeridos, de forma a relacionar a dieta com estado nutricional dos indivíduos, utilizam-se instrumentos como o Recordatório de 24 horas, o Registro de Ingestão Alimentar e o Questionário de Frequência Alimentar. ${ }^{10}$ Além disso, vários índices vêm sendo propostos para quantificar o padrão de qualidade da dieta, como o Índice de Qualidade de Dieta (IQD), por meio do qual se avalia a ingestão adequada de nutrientes, número de porções consumidas de carboidratos, frutas e hortaliças, a quantidade de gordura total, saturada, colesterol, proteína, cálcio e sódio ingeridos pelo indivíduo. ${ }^{11}$ Assim, por meio do IQD é possível avaliar o quanto a dieta está adequada e saudável, ${ }^{11}$ baseando-se nas recomendações da Diet and Health. ${ }^{12}$

A avaliação bioquímica é de extrema importância, pois permite complementar a avaliação do exame físico, dados antropométricos e de ingestão alimentar, contribuindo para um melhor diagnóstico. ${ }^{13}$ Os exames mais comumente usados são perfil lipídico (colesterol total, HDL-c, LDL-c 
e triacilgliceróis) e glicemia, já que estes parâmetros tendem a estar alterados no excesso de peso e caracterizar a presença de síndrome metabólica. ${ }^{14}$

Perante aos expostos acima, o objetivo desse trabalho foi avaliar o estado nutricional por meio das medidas antropométricas, de composição corporal e bioquímicas, bem como verificar a associação destes parâmetros com o IQD e os componentes de síndrome metabólica em indivíduos com excesso de peso.

\section{Material e Métodos}

O estudo do tipo transversal, com amostra não probabilística por conveniência, foi realizado no Ambulatório de Nutrição localizado no Centro de Saúde da Universidade Federal de Ouro Preto, no período entre 2011 a 2013. Os pacientes que buscavam atendimento dietoterápico eram informados sobre a pesquisa e selecionados após assinarem o Termo de Consentimento Livre e Esclarecido.

Para serem incluídos, os voluntários, independente do sexo, deveriam possuir idade superior a 18 anos e diagnóstico nutricional de sobrepeso ou obesidade pelo IMC. Excluíram-se as crianças, os adolescentes e os indivíduos com diagnóstico nutricional de eutrofia ou baixo peso.

Os voluntários foram submetidos a uma avaliação antropométrica (peso, estatura, circunferências e pregas cutâneas), dietética (Registro alimentar) e bioquímica (hemograma completo, perfil lipídico e glicose). O peso corporal foi aferido utilizando-se a balança eletrônica da marca WELMY® com capacidade para $150 \mathrm{~kg}$. A estatura foi mensurada através do estadiômetro de metal acoplado à balança eletrônica, com precisão de $0,1 \mathrm{~mm}$. Para ambas as medidas, os voluntários estavam descalços, com roupas leves, em posição ereta no centro do equipamento.

A medida das pregas cutâneas tricipital (PCT-mm), bicipital (PCB-mm), subescapular (PCSE$\mathrm{mm}$ ) e suprailíaca (PCSI-mm), foi realizada utilizando um adipômetro da marca Cescorf que possui pressão de $10 \mathrm{~g} / \mathrm{mm}^{2}$, sensibilidade de $0,1 \mathrm{~mm}$ e amplitude de leitura de $85 \mathrm{~mm}$. O valor anotado foi a média das 3 medidas consecutivas. ${ }^{10}$

A aferição das circunferências abdominal (CA-cm), braquial (CB-Cm), da cintura (CC-cm) e do quadril (CQ-cm) foi realizada com fita métrica inelástica, com precisão de $0,1 \mathrm{~cm}$. A CA foi coletada no ponto médio em cima da cicatriz umbilical, já a CC no ponto médio entre a última costela e a crista ilíaca (na curvatura natural). A CB foi aferida entre o ponto médio do acrômio e o olecrano com o indivíduo com o braço estendido ao longo do corpo. Para a CQ, a fita métrica flexível circundou o quadril na região de maior perímetro entre a cintura e a coxa, com o indivíduo usando roupas finas e leves. ${ }^{15}$

Os dados antropométricos foram utilizados no cálculo do IMC $\left(\mathrm{Kg} / \mathrm{m}^{2}\right)$, da relação cinturaquadril (RCQ - cm), da circunferência muscular do braço (CMB-cm), do percentual de gordura 
corporal (\%CG) e do índice ateratogênico. A RCQ o indicador mais comumente utilizado para identificar a distribuição de gordura corporal, foi determinada por meio da equação: $\mathrm{RCQ}=$ CC/ CQ. Já a CMB, que avalia a reserva de tecido muscular, foi obtida pelos valores de CB e PCT pela fórmula: $\mathrm{CMB}(\mathrm{cm})=\mathrm{CB}(\mathrm{cm})-\Pi \mathrm{x}[\mathrm{PCT}(\mathrm{mm}) / 10]$. O \%GC foi obtido pelo somatório das 4 pregas cutâneas e o índice aterogênico pela divisão da PCSE pela PCT. ${ }^{15}$

A classificação do estado nutricional dos indivíduos com excesso de peso foi realizada conforme os pontos de corte que considera IMC entre 25,0 e $29,9 \mathrm{~kg} / \mathrm{m}^{2}$ (sobrepeso ou pré-obesidade), IMC entre 30,0 e $34,9 \mathrm{~kg} / \mathrm{m}^{2}$ (obesidade grau I), IMC entre 35,0 e $39,9 \mathrm{~kg} / \mathrm{m}^{2}$ (obesidade grau II) e IMC $\geq 40,0 \mathrm{~kg} / \mathrm{m}^{2}$ (obesidade grau III). ${ }^{10}$

Foram solicitados os seguintes exames laboratoriais: hemograma completo; perfil lipídico (colesterol total, LDL-c, HDL-c e triacilgliceróis) e glicose. Estes exames foram determinados no Laboratório Piloto de Análises Clínicas da Universidade Federal de Ouro Preto (LAPAC//UFOP). Para a realização do exame bioquímico, os voluntários foram orientados a permanecer em jejum por 8 horas.

Posteriormente avaliou-se a ingestão dietética por meio de recordatório de 24 horas, onde o voluntário relatava os alimentos e bebidas ingeridos no dia anterior; e registro alimentar de 72 horas no qual o indivíduo era instruído a registrar no momento do consumo, os alimentos e bebidas consumidos em dias alternados, contemplando dois dias da semana (dias típicos) e um dia do fim de semana (dia atípico). ${ }^{10}$

Os dados de ingestão foram digitados no software Dietwin para a quantificação dos macronutrientes (carboidratos, proteínas e lipídeos). A avaliação da qualidade da dieta foi feita por meio da pontuação do IQD, onde o consumo de frutas e verduras, carboidratos, proteínas, lipídeos totais, saturados, colesterol, sódio e cálcio foi avaliado através de um escore que varia de 0 a $2{ }^{11}$ de acordo com a recomendações da Diet and Healt. ${ }^{12}$ A partir desses parâmetros, o indivíduo podia apresentar uma pontuação 0 que indica uma dieta de excelente qualidade, ou no atingir no máximo os 16 pontos, sendo sua ingestão considerada como péssima. ${ }^{11}$

A partir dos dados antropométricos e bioquímicos coletados, os indivíduos foram classificados quanto à presença de síndrome metabólica, adotando-se o critério de associação de pelo menos 3 fatores de risco: obesidade abdominal, dislipidemia aterogênica (diminuição do HDL-c e aumento do LDL-c), hipertrigliceridemia, hipertensão arterial sistêmica, hiperglicemia. ${ }^{16}$ Após todas as avaliações, os indivíduos receberam orientações nutricionais individuais pelos acadêmicos de nutrição devidamente treinados.

As análises estatísticas foram realizadas utilizando o programa Predictive Analytics Software (PASW) Statistics 18 considerando o nível de significância 5\%. ${ }^{17}$ Utilizou-se o teste de KolmogorovSmirnov para testar a normalidade dos dados. As variáveis de distribuição normal foram 
apresentadas em média e desvio padrão, enquanto que, as de distribuição não normal em mediana e intervalo interquartil. A correlação de Pearson foi utilizada a fim de verificar a associação entre os componentes da síndrome metabólica com os dados antropométricos, bioquímicos e de composição corporal, bem como a associação dos componentes do IQD com medidas antropométricas, de composição corporal, clínicas e bioquímicas dos voluntários. O teste Exato de Fisher foi realizado para analisar os componentes do IQD com a presença ou ausência de síndrome metabólica.

O estudo foi aprovado pelo Comitê de Ética em Pesquisa da Universidade Federal de Ouro Preto, em Oficio CEP №073/2011.

\section{Resultados e Discussão}

O estudo contou com a participação de 68 voluntários, sendo 27,94\% (n=19) do sexo masculino e $72 \%$ (n=49) do sexo feminino, com idade entre 20 e 57 anos. Foi analisada a frequência de síndrome metabólica (SM) nessa população, já que a obesidade e excesso de peso vêm sendo apontados como fundamentais na gênese dessa síndrome. ${ }^{16,18}$ Dos indivíduos acompanhados 16,17\% $(n=11)$ apresentaram SM, ou seja, possuem fatores usualmente relacionados à deposição central de gordura e a resistência à insulina. Dados semelhantes foram encontrados por Pimenta et al., ${ }^{19}$ que avaliaram a prevalência de SM e seus fatores associados em uma área rural de Minas Gerais, observando uma prevalência $14,9 \%$.

Ressalta-se que a SM vem sendo considerada do ponto de vista epidemiológico, como responsável pelo aumento da mortalidade cardiovascular estimada em 2,5 vezes. Neste aspecto, é importante destacar que vários estudos têm investigado a associação entre obesidade e SM, contudo o não estabelecimento dos fatores de risco considerados e a divergência entre os critérios diagnósticos têm inviabilizado a sua comparação. No Brasil não foram encontrados dados representativos sobre a prevalência da SM, entretanto, estudos em diferentes populações, como a mexicana, a norte americana e a asiática, demonstram prevalências elevadas, dependendo do critério utilizado e das características da população, variando as taxas de 12,4\% a 28,5\% em homens e de 10,7\% a 40,5\% em mulheres. ${ }^{16}$

A tabela 1 apresenta as medidas antropométricas, de composição corporal, bioquímicas e dietéticas. Os indivíduos acompanhados apresentaram excesso de adiposidade, confirmado pela a média do IMC, CC, RCQ, \%GC e pelas 4 pregas cutâneas, o que já era esperado, devido ao critério de inclusão dos voluntários, que deveriam apresentar excesso de peso. Em nosso estudo a média do IMC

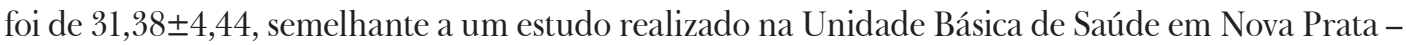
RS, em que esse valor foi de $32,83 \mathrm{~kg} / \mathrm{m} .{ }^{20}$ Podemos ressaltar que a CC vem sendo considerada como um ótimo indicador de distribuição corporal e risco de desenvolver outras morbidades. ${ }^{21}$ Além disso, a distribuição corporal dependendo da sua localização predispõe o individuo a um maior risco de complicações metabólicas, sendo a RCQ o indicador mais comumente usado para esta classificação. ${ }^{15}$ 


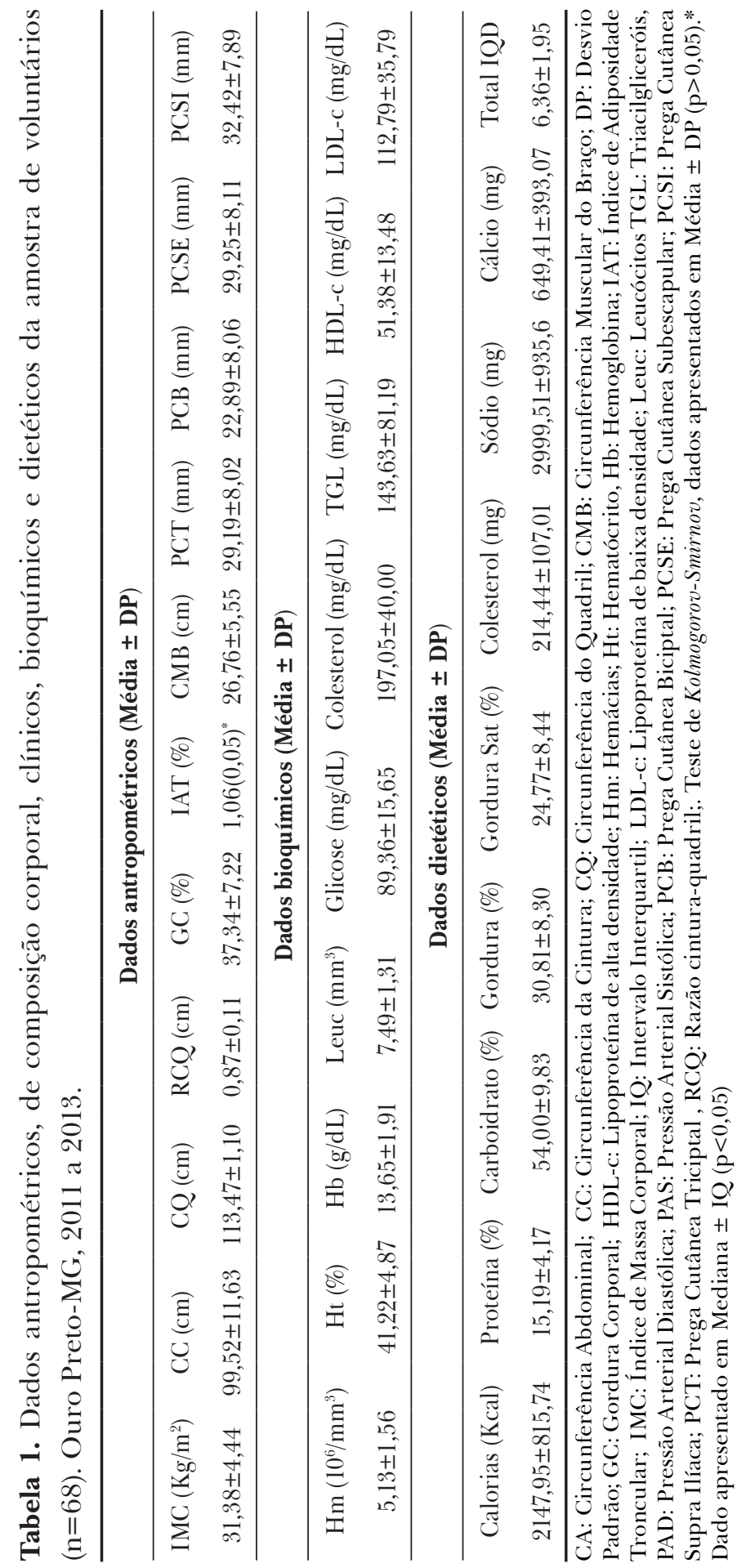


A obesidade associa-se ao surgimento de várias comorbidades, como resistência à insulina, diabetes mellitus tipo 2, dislipidemias e hipertensão arterial. ${ }^{10,15}$ Em relação aos dados bioquímicos, as médias de glicose e colesterol total mantiveram-se dentro dos valores de referência, contudo o LDL-c esteve na faixa considerada limítrofe $(<129 \mathrm{mg} / \mathrm{dL})$ e HDL-c abaixo da recomendação ( $\geq$ $60 \mathrm{mg} / \mathrm{dL}){ }^{22}$ Esse perfil bioquímico em indivíduos com excesso de peso é encontrado na literatura, como no estudo de Ramos et al., ${ }^{23}$ que avaliou o perfil socioeconômico, antropométrico, bioquímico e estilo de vida de pacientes atendidos no programa "controle de peso", demonstrando que o LDL-c estava acima do recomendado e o HDL-c abaixo do valor de referência.

No que tange a pontuação do IQD, o valor médio encontrado foi de 6,36 $\pm 1,98$ para os homens e 5,59 $\pm 2,24$ para as mulheres, inferindo qualidade da dieta mais próxima do ideal. O consumo dos macronutrientes (lipídeos, proteínas e carboidratos) esteve dentro da faixa de recomendação das DRIs (Dietary Reference Intakes), ${ }^{24}$ o que é muito importante, uma vez que o consumo inadequado está relacionado com a gênese da obesidade. ${ }^{5}$ Ao contrário dos dados encontrados, evidências sugerem um consumo inadequado de macronutrientes na obesidade, como os pacientes atendidos em uma clínica de Porto Alegre-RS, que apresentaram um alto consumo de carboidratos, lipídeos e proteínas. ${ }^{25}$ Já uma pesquisa que avaliou a qualidade da dieta dos funcionários de uma universidade pública, por meio do índice de alimentação saudável, observou um alto consumo de lipídeos. ${ }^{26}$

Nota-se que o consumo de sódio está acima do recomendado e cálcio abaixo da recomendação. O aumento do consumo de sódio juntamente com a obesidade, são fatores predisponentes do surgimento da hipertensão arterial, ${ }^{27}$ entretanto os indivíduos avaliados apresentaram a média

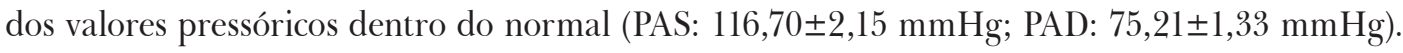
Diferentemente dos nossos resultados, Manfroi et al., ${ }^{28}$ avaliando o consumo de sódio em pacientes com síndrome metabólica, encontraram que $81.8 \%$ dos indivíduos analisados apresentavam pelo menos uma das pressões elevadas, e valores médios de consumo de sódio abaixo do recomendado (1483 $\pm 777,9$ mg). Já Sarno et al., ${ }^{29}$ demonstraram que a quantidade de consumo brasileiro de sódio excede em mais de duas vezes o limite de ingestão recomendado, corroborando os nossos dados. O consumo excessivo de sódio também ocorre em países desenvolvidos, como demonstra o estudo realizado por $\mathrm{COOK}^{30}$ nos Estados Unidos, que evidencia um consumo médio de $3600 \mathrm{mg}$ por dia.

Em relação ao cálcio, é evidenciado sua participação na fisiopatologia da obesidade, pois quando ingerido abaixo da recomendação, induz ao aumento da lipogênese, diminuição da lipólise e oxidação lipídica. Outro fato relevante é que o cálcio pode associar a ácidos graxos livres no trato gastrointestinal, diminuindo sua absorção. ${ }^{31}$ Assim atingir o consumo adequado de cálcio torna-se uma meta fundamental para os indivíduos acompanhados.

Quando analisado a correlação dos componentes da síndrome metabólica com os dados antropométricos, bioquímicos e de composição corporal, encontramos correlação negativa entre 
IMC e HDL-c $(p=0,002)$, e correlação positiva entre \% GG e triglicérides $(p=0,001)$ (Tabela 2). Rezende et al., ${ }^{32}$ também observou redução do HDL-c em decorrência do aumento do IMC e da gordura abdominal. Em contrapartida, houve aumento triglicérides devido à elevação da gordura corporal, IMC e CA. O excesso de peso juntamente com a redução do HDL-c e aumento dos triglicerídeos, são fatores de risco importante para o surgimento de complicações cardiovasculares, sendo fundamental o acompanhamento nutricional a fim de reduzir o IMC e o \%GC. ${ }^{16}$

Tabela 2. Correlações significativas dos componentes da síndrome metabólica com parâmetros antropométricos da amostra de voluntários (n=68), Ouro Preto-MG, 2011-2013.

\begin{tabular}{cccccccc}
\hline \multirow{2}{*}{ Variáveis } & \multicolumn{7}{c}{ Componentes da síndrome metabólica } \\
\cline { 2 - 8 } & Glicose & $\begin{array}{c}\text { PAS } \\
(\mathrm{mmHg})\end{array}$ & $\begin{array}{c}\text { PAD } \\
(\mathrm{mmHg})\end{array}$ & CA (cm) & $\begin{array}{c}\text { Triglicérides } \\
(\mathrm{mg} / \mathrm{dL})\end{array}$ & $\begin{array}{c}\text { LDL-c } \\
(\mathrm{mg} / \mathrm{dL})\end{array}$ & $\begin{array}{c}\text { HDL-c }(\mathrm{mg} / \\
\mathrm{dL})\end{array}$ \\
\hline IMC $\left(\mathrm{kg} / \mathrm{m}^{2}\right)$ & $\mathrm{r}=0,052$ & $\mathrm{r}=0,073$ & $\mathrm{r}=0,430$ & $\mathrm{r}=0,011$ & $\mathrm{r}=0,026$ & $\mathrm{r}=0,003$ & $\mathrm{r}=\mathbf{- 0 , 4 3 4 * *}$ \\
& $\mathrm{p}=0,065$ & $\mathrm{p}=0,227$ & $\mathrm{p}=0,455$ & $\mathrm{p}=0,227$ & $\mathrm{p}=0,878$ & $\mathrm{p}=0,959$ & $\mathbf{p = 0 , 0 0 2}$ \\
& & & & & & & \\
IAT (\%) & $\mathrm{r}=0,118$ & $\mathrm{r}=0,124$ & $\mathrm{r}=0,457$ & $\mathrm{r}=0,714$ & $\mathrm{r}=0,133$ & $\mathrm{r}=0,313$ & $\mathrm{r}=0,174$ \\
& $\mathrm{p}=0,150$ & $\mathrm{p}=0,570$ & $\mathrm{p}=0,679$ & $\mathrm{p}=0,172$ & $\mathrm{p}=0,127$ & $\mathrm{p}=0,089$ & $\mathrm{p}=0,087$ \\
GC (\%) & $\mathrm{r}=0,674$ & $\mathrm{r}=0,284$ & $\mathrm{r}=0,382$ & $\mathrm{r}=0,340$ & $\mathbf{r}=\mathbf{0 , 5 6 1 * *}$ & $\mathrm{r}=0,332$ & $\mathrm{r}=0,139$ \\
& $\mathrm{p}=0,312$ & $\mathrm{p}=0,122$ & $\mathrm{p}=0,654$ & $\mathrm{p}=0,567$ & $\mathbf{p = 0 , 0 0 1}$ & $\mathrm{p}=0,701$ & $\mathrm{p}=0,141$ \\
\hline
\end{tabular}

CA: Circunferência abdominal; GC: Gordura Corporal; IAT: Índice de Adiposidade Troncular; IMC: Índice de Massa Corporal; LDL-c: Lipoproteína de baixa densidade; PAS: Pressão arterial sistólica; PSD: Pressão arterial diastólica; r: Coeficiente de correlação de Pearson. * $\mathrm{p}<0,05, * * \mathrm{p}<0,01$.

Não houve correlação positiva entre a glicemia e os parâmetros antropométricos, o que não era esperado, já que o excesso de peso muitas vezes se associa ao surgimento de resistência à insulina, hiperglicemia e diabetes mellitus tipo II. ${ }^{14,22}$

Observa-se que houve correlação positiva entre a CQ com o cálcio e colesterol com o IQD (Tabela 3). A associação inversa entre consumo de cálcio e aumento do peso corporal tem sido relatada, sendo proposto que o cálcio altera a absorção de gordura pelo organismo e afeta o metabolismo dos adipócitos. ${ }^{31}$ Esteves et al., ${ }^{33}$ não encontraram correlação significativa entre as variáveis de adiposidade analisadas (IMC, \%GC, CC e RCQ) e a ingestão de cálcio. Contrariando esse achado, Zemel $^{34}$ demonstraram que as mulheres com ingestão de $1300 \mathrm{mg} /$ dia de cálcio possuem menor risco de acumular gordura corporal. 


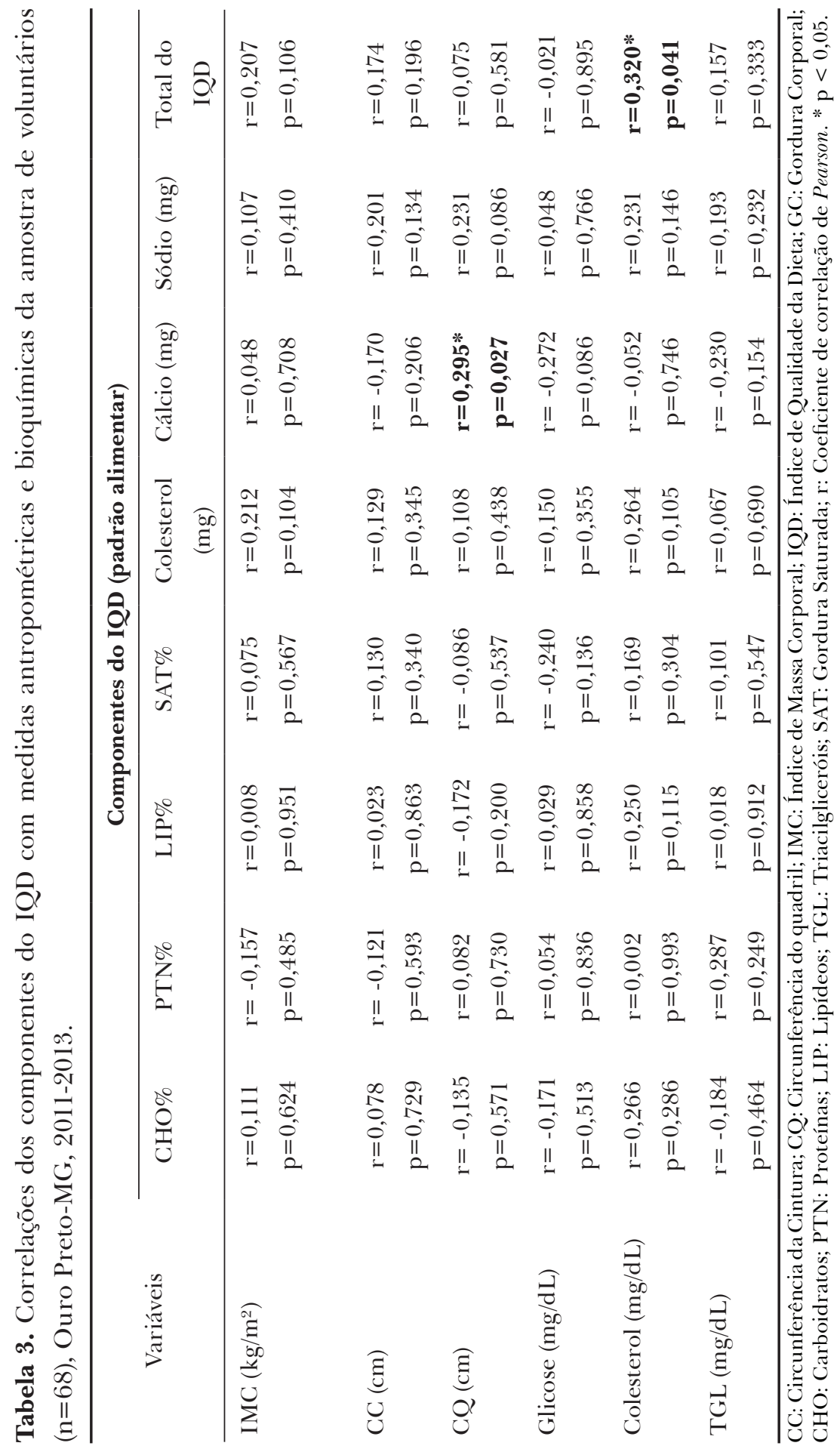


Em relação ao colesterol, não foram encontrados até o presente momento, dados que o correlacionem com o IQD. Provavelmente a correlação encontrada pode ser explicada pelo fato de o aumento do colesterol estar associado a hábitos dietéticos inadequados, ${ }^{10}$ que por sua vez relaciona-se ao aumento da pontuação do IQD. ${ }^{11}$

Por fim, a tabela 4 evidencia que não houve associação entre os componentes do IQD e presença ou ausência de SM, contrariando ao que vêm sendo encontrado na literatura, como no artigo de revisão de Steemburgo et al., ${ }^{35}$ o qual aponta várias pesquisas demonstrando a importância dos componentes da dieta na gênese e proteção da SM.

Tabela 4. Análise dos componentes do IQD com presença e ausência de síndrome metabólica, Ouro Preto - MG, 2011-2013.

\begin{tabular}{|c|c|c|c|c|c|}
\hline \multirow{3}{*}{ Componentes do IQD } & \multicolumn{4}{|c|}{ Indivíduos } & \\
\hline & \multicolumn{2}{|c|}{$\begin{array}{l}\text { Ausência de } \\
\text { síndrome } \\
\text { metabólica }\end{array}$} & \multicolumn{2}{|c|}{$\begin{array}{c}\text { Presença de } \\
\text { síndrome } \\
\text { metabólica }\end{array}$} & \\
\hline & $\mathrm{N}$ & $\%$ & $\mathrm{~N}$ & $\%$ & p-valor \\
\hline
\end{tabular}

\section{Lipídeos totais}

$\leq 30 \%$
$30-40 \%$
$>40 \%$
Ácidos graxos saturados

$\leq 10 \%$

32

28

49,10

5

45,50

$10-13 \%$

14

33,33

5

45,50

0,260

$>13 \%$

6

12,30

0

0,00

Colesterol

$$
\begin{aligned}
& \leq 300 \mathrm{mg} \\
& 300-400 \mathrm{mg} \\
& >400 \mathrm{mg}
\end{aligned}
$$

66,70

6

54,50

$8 \quad 14,00$

2

18,20

0,344

6

10,50

1

9,10 


\begin{tabular}{l|l|l|c|c|c}
\hline \multirow{2}{*}{ Componentes do IQD } & \multicolumn{4}{|c|}{ Indivíduos } & \\
\cline { 2 - 5 } & \multicolumn{2}{|c|}{$\begin{array}{c}\text { Ausência de } \\
\text { síndrome } \\
\text { metabólica }\end{array}$} & \multicolumn{2}{|c|}{$\begin{array}{c}\text { Presença de } \\
\text { síndrome } \\
\text { metabólica }\end{array}$} & \\
\cline { 2 - 6 } & $\mathrm{N}$ & $\%$ & $\mathrm{~N}$ & $\%$ & $\mathrm{p}$-valor \\
\hline
\end{tabular}

\section{Hortaliças/verduras/frutas}

$5 \mathrm{ou}+$ porções

3-4 porções

0-2 porções

Pães, grãos e cereais

$6 \mathrm{ou}+$ porções

4-5 porções

0-3 porções

\section{Proteína}

$\leq 100 \% \mathrm{RDA}$

100-150\% RDA

$>150 \% \mathrm{RDA}$

\section{Sódio}

$\leq 2400 \mathrm{mg}$

2400-3400mg

$>3400 \mathrm{mg}$

\section{Cálcio}

$\geq \mathrm{RDA}$

2/3 RDA

$<2 / 3$ RDA
7

9

24

12,30

15,80

42,10

24

42,10

19,30

8,80

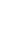

0

36,40

0,556

5

21

36,80

3

27,30

23

40,40

5

45,50

0,440

9

15,80

2

18,20

23

40,40

2

18,20

17

29,80

7

63,60

0,120

13

22,80

1

9,10

IQD: Índice de Qualidade da Dieta. $\mathrm{p}>0,05$ para todas as variáveis analisadas - Teste Exato de Fisher. RDA: Recommended Dietary Allowances ${ }^{24}$ 


\section{Conclusão}

Os resultados demonstram que os indivíduos com excesso de peso corporal possuem um maior risco de desenvolver morbidades, já que apresentaram alterações antropométricas, bioquímicas e ingestão inadequada de cálcio e de sódio. Assim, nota-se a importância do acompanhamento nutricional, de modo a evitar agravos a saúde e a conscientizar esses pacientes sobre a importância de uma alimentação saudável, atuando no controle e prevenção das complicações encontradas, evitando novas ocorrências de síndrome metabólica.

\section{Agradecimentos}

À Universidade Federal de Ouro Preto e à Escola de Nutrição, pela viabilidade e apoio a pesquisa; e a todos os voluntários que gentilmente participaram do estudo.

\section{Referências}

1. Chantal J, Ducrot P, Lassale C, Fézeu L, Méjean C, Péneau S, et al. Prospective associations between a dietary index based on the British Food Standard Agency nutrient profiling systemand 13-yearweight gain in the SU.VI.MAX cohort. Preventive Medicine 2015; 81:189-94.

2. Byker Shanks C, Smith T, Ahmed S, Hunts H. Assessing Foods offered in the Food Distribuition Program on Indian Reservations (FDPIR) using the Healthy Eating Index 2010. Public Health Nutr. 2016; 19(7):1315-26.

3. World Health Organization Obesity and overweight. Fact Sheet, no. 311. January 2015. [acesso em: 22 set. 2015]. Disponível em: http://www.who.int/mediacentre/factsheets/fs311/en/

4. Brasil. Ministério da Saúde. Vigitel 2013: vigilância de fatores de risco e proteção para doenças crônicas por inquérito telefônico. Brasília: Ministério da Saúde; 2014. 121 p. [acesso em: 02 jan. 2015]. Disponível em: https://biavati.files.wordpress.com/2014/05/vigitel-2013.pdf

5. Carrara APB, França EA, Bonino MV, Brochetto MFD, Ribeiro RL, Costa ECS, et al. Obesidade: um desafio para a saúde pública. Rev. Inst. Ciênc. Saúde 2008; 26(3):299-303.

6. Malta DC, Morais Neto OL, Silva Junior JB. Apresentação do plano de ações estratégicas para o enfrentamento das doenças crônicas não transmissíveis no Brasil, 2011 a 2020. Epidemiol. Serv. Saúde 2011; 20(4):425-438.

7. Kanno P, Rabelo M, Melo GF, Giavoni A. Discrepâncias na imagem corporal e na dieta de obesos. Rev. Nutr. 2008; 21(4):423-430.

8. Sales RL, Silva MMS, Costa NMB, Euclydes MP, Eckhardt VF, Rodrigues ALA, et al. Desenvolvimento de um inquérito para avaliação da ingestão alimentar de grupos populacionais. Rev. Nutr. 2006; 19(5):539-552. 
9. Buzzachera CF, Krause MP, Elsangedy HM, Hallage T, Granato P, Krinski K, et al. Prevalência de sobrepeso e obesidade geral e central em mulheres idosas da cidade de Curitiba, Paraná. Rev. Nutr. 2008; 21(5):525-533.

10. MahaM LK, Escott-Stump S. Krause alimentos, nutrição e dietoterapia. 12 ed. Rio de Janeiro: Elsevier; 2011.

11. Volp ACP, Alfenas RCG, Costa NMB, Minim VPR, Stringueta PC, Bressan J. Índices dietéticos para avaliação da qualidade de dietas. Rev. Nutr. 2010; 23(2):281-295.

12. National Research Council. Diet and health: implications for reducing chronic diseases risk. Washington (DC): National Academy of Sciences; 1989.

13. Gaiano NM, Silva MV. Consumo de frutoses e impacto na saúde humana. Rev. Segurança Alimentar e Nutricional 2011; 18(2):86-98.

14. Fett CA, Fett WCR, Marchini JS. Comparação entre bioimpedância e antropometria e a relação dos índices corporais ao gasto energético de repouso e marcadores bioquímicos sanguíneos em mulheres da normalidade à obesidade. Rev. Bras. Cineantropom. Desempenho Hum. 2006; 8(1):29-36.

15. Cuppari L. Guias de medicina ambulatorial e hospitalar: nutrição clinica no adulto. $3^{a}$ ed. Barueri, SP: Manole; 2014.

16. Sociedade Brasileira de Cardiologia. I Diretriz brasileira de diagnóstico e tratamento da síndrome metabólica. Arquivos Brasileiros de Cardiologia 2005; 84(Supl. 1):1-28.

17. PASW Statistics 18 [Internet]. [acesso em: 24 set. 2015]. Disponível em: http://www.spss.com.hk/ statistics/

18. Paccini MK, Arsa G, Glaner MF. Indicadores de gordura abdominal: antropometria vs absortometria de raio-x de dupla energia. Revista Brasileira de Cineantropometria \& Desempenho Humano 2008; 10(3): 283-288.

19. Pimenta AM, Gazzinelli A, Veláquez-Meléndez G. Prevalência da síndrome metabólica e seus fatores associados em área rural de Minas Gerais (MG, Brasil). Ciência \& Saúde Coletiva 2011; 16(7):3297-3306.

20. Silva PC, Zaffari D. Prevalência de excesso de peso e associação com outras variáveis em indivíduos adultos atendidos em unidade básica de saúde. Scientia Medica 2009; 19(1):17-26.

21. Martins IS, Marinho SP. O pontencial diagnóstico dos indicadores da obesidade centralizada. Rev. Saúde Pública 2003; 37(6):760-7.

22. Nemer ASA; Neves FJ, Ferreira JES. Manual de solicitação e interpretação de exames laboratoriais. Rio de Janeiro: Revinter; 2010.

23. Ramos JN, Damacena LC, Stringhini MLF, Fornés NS. Perfil socioeconômico, antropométrico, bioquímico e estilo de vida de pacientes atendidos no programa "controle de peso". Comun. Ciênc. Saúde 2006; 17(3):185-92.

24. Institute of Medicine. Dietary references intakes for energy, carbohydrate, fiber, fat, fatty acids, cholesterol, protein and amino acids. Washington, DC: National Academic Press; 2005. 
25. Sauer P, Oliveira KS, Carvalho L, Ferrano JLS, Alves MK. Perfil alimentar de pacientes com sobrepeso e obesidade atendidos em uma unidade clínica de Porto Alegre entre os anos de 2000 e 2006. X Salão de Iniciação Científica PUCRS; 2009; 881-83.

26. Nespeca M, Cyrillo DC. Avaliação da qualidade da dieta por meio do índice de alimentação saudável de funcionários de uma universidade pública. Rev. Soc. Bras. Alim. Nutr. 2010; 35(2):81-90.

27. Sociedade Brasileira de Cardiologia. VI diretrizes brasileiras de hipertensão. Arq Bras Cardiol. 2010; 95(Supl. 1):1-51.

28. Manfroi GF, Santos RS, Teixiera AS, Feoli AMP, Silva VL. Consumo de sódio em usuários com síndrome metabólica de uma unidade de saúde. Rev. Grad. 2009; 2(7):1-17.

29. Sarno F, Claro RM, Levy RB, Bandoni DH, Ferreira SRG, Monteiro CA. Estimativa de consumo de sódio pela população brasileira, 2002-2003. Rev. Saúde Pública 2009; 43(2):219-25.

30. Cook NR. Salt intake, blood pressure and clinical outcomes. Current Opinion in Nephrology and Hypertension 2008; 17(3):310-314.

31. Silva PMC, Cabral Junior CR, Vasconcelos SML. Ingestão do cálcio na obesidade de mulheres atendidas pelo Sistema Único de Saúde. Rev. Nutr. 2010; 23(3):357-367.

32. Rezende FAC, Rosado LEFPL, Ribeiro RCL, Vidigal FC, Vasques ACJ, Bonard IS, et al. Índice de Massa Corporal e Circunferência da Abdominal; associação com fatores de risco cardiovascular. Arq. Bras. Cardiol. 2006; 87(6):728-734.

33. Esteves EA, Rodrigues CAA, Paulino EJ. Ingestão dietética de cálcio e adiposidade em mulheres adultas. Rev. Nutr. 2010; 23(4):543-552.

34. Zemel MB, Shi H, Greer B, Dirienzo D, Zemel PC. Regulation of adiposity by dietary calcium. Faseb J. 2000; 14(9):1132-8.

35. Stemburgo T, Dall'Alba V, Gross JL, Azevedo MJ. Fatores dietéticos e síndrome metabólica. Arquivos Brasileiros de Endocrinologia e Metabologia 2007; 51(9):1425-1433.

Recebido: $14 / 3 / 2015$

Revisado: $22 / 9 / 2015$

Aceito: 04/2/2016 
